\title{
Oscillate Criteria of Third Order Semi-linear Neutral Delay Differential Equations
}

\author{
Lin Jingjie ${ }^{1}$, Zheng Liangtian ${ }^{1} \&$ Lin Quanwen ${ }^{1}$ \\ ${ }^{1}$ Department of Mathematics and Applied Mathematics, Science of School, Guangdong University of Petrochemical \\ Technology, Maoming Guangdong, China \\ Correspondence: Lin Quanwen,Guandu Second Road, Department of Mathematics, Scienceof School, Guangdong \\ University of Petrochemical Technology, Maoming of Guangdong China. E-mail: linquanwen@ 126.com
}

Received: March 3, 2020 Accepted: March 26, 2020 Online Published: April 26, 2020

doi:10.5539/jmr.v12n3p23

URL: https://doi.org/10.5539/jmr.v12n3p23

\section{Abstract}

The oscillation of a class of neutral third-order semi-linear differential equations is studied. The Riccati transform technique is used to construct different functions and classical inequalities. Some new oscillation theories of differential equations are established. Our results differ from the results in other literature, and use examples to illustrate the application of the conclusions.

Keywords: third-order neutral differential equation, semi-linear, oscillation, Riccati transformation

\section{Introduction}

Consider the oscillation of a class of third-order semilinear neutral delay differential equations of the form

$$
\left[r(t)\left|Z^{\prime \prime}(t)\right|^{\alpha-1} Z^{\prime \prime}(t)\right]+q(t)|x(\sigma(t))|^{\beta-1} x(\sigma(t))=0, t \geq t_{0}>0 . \beta<\alpha
$$

Where $Z(t)=x(t)+p(t) x(\tau(t)), \beta>0, \alpha>0, \alpha, \beta$ are the quotients of two positive and odd integers.

Assume the following conditions hold

$\left(A_{1}\right) p(t), q(t) \in C\left(\left[t_{0}, \infty\right),(0, \infty)\right), 0 \leq p(t) \leq p<1, q(t)>0 ;$

$\left(A_{2}\right) \quad r(t) \in C^{1}\left(\left[t_{0}, \infty\right),(0, \infty)\right), r(t) \geq 0, r^{\prime}(t) \geq 0, \int_{t_{0}}^{\infty} r^{-\frac{1}{\alpha}}(s) d s \leq+\infty$;

$\left(A_{3}\right) \tau(t), \sigma(t) \in C^{1}\left(\left[t_{0}, \infty\right),(0, \infty)\right)$, for each $t \geq t_{0}$, there is $\mathrm{b} \tau(t) \leq t, \sigma(t) \leq t, \sigma(t)>0, \sigma^{\prime}(t)>0$,

$\lim _{t \rightarrow \infty} \tau(t)=\lim _{t \rightarrow \infty} \sigma(t)=\infty$.

According to the custom, the solution of the equation is called oscillatory, if it has arbitrarily large zeros; otherwise it is said to be non- oscillatory. If all the solutions of the equation are oscillatory, then the equation is called oscillatory; otherwise it is called non- oscillatory.

References (X. X. SU, L. N. Dai, S. M. Wu., \& Q. W. LIN,2017, H. LIU, F. MENG, \& P. LIU, 2012, and ZENG, Y. H., LUO, L. P., \& YU, Y. H, 2015) for second-order semi-linear neutral differential equations

$$
\left(r(t)\left|Z^{\prime}(t)\right|^{\alpha-1} Z^{\prime}(t)\right)^{\prime}+q(t)|x(\sigma(t))|^{\beta-1} x(\sigma(t))=0 .
$$

n-depth research was done to give some new oscillate criteria. In the past few years, the research on the vibration of third-order semi-linear differential equations has begun to attract attention, but its research results on oscillations are still relatively small, such as references(LI, Y. D., GAO ZH. H., \& DENG Y. H,2012), and References fifth to fifteen. In 2017, Hui Yuanxian et al. Established a number of new oscillate criteria to guarantee that all solutions of the equation 
(E) oscillate or converge to zero under the condition of limiting $\int_{t_{0}}^{\infty} r^{-\frac{1}{\alpha}}(s) d s=+\infty$.

Recently, Ref. (S. M. WU, J. J. LIN, Q. D. LI., \& Q. W. LIN,2019) studied several new vibrational criteria for all solutions of the equation $\left[r(t)\left|Z^{\prime \prime}(t)\right|^{\alpha-1} Z^{\prime \prime}(t)\right]+q(t)|x(\sigma(t))|^{\beta-1} x(\sigma(t))=0$,

to vibrate or converge to zero in the case of $t \geq t_{0}>0, \beta>\alpha$.

Inspired by the work in References (SU, et al 2017), and References fourteenth and fifteenth, the Riccati transformation and classical inequalities were used to establish a new conclusion for the oscillation of the equation under the conditions $\int_{t_{0}}^{\infty} r^{-\frac{1}{\alpha}}(s) d s \leq+\infty$ and $\beta<\alpha$, which generalized and improved Some results are given, and some examples are given to illustrate the application of the main results.

\section{Lemma}

Lemma2.1 (HUI, Y. X., \& WANG, J. J., 2017). If $x(t)$ is the final positive solution of equation $(E)$, then $Z(t)$ has only the following two possibilities, that is, there is $T \geq t_{0}$, so that when $t \geq T$, there are

(A) $Z(t)>0, Z^{\prime}(t)>0, Z^{\prime \prime}(t)>0$.

(B) $Z(t)>0, Z^{\prime}(t)<0, Z^{\prime \prime}(t)>0$.

Lemma 2.2(WU, et al, 2019) If $A>0, B>0$ and $\alpha>0$ exist, then $B u-A u^{\frac{\alpha+1}{\alpha}} \leq \frac{\alpha^{\alpha}}{(\alpha+1)^{\alpha+1}} \frac{B^{\alpha+1}}{A^{\alpha}}$.

Lemma 2.3(WU, et al, 2019) Let $u(t)>0, u^{\prime}(t)>0, u^{\prime \prime}(t) \leq 0, t \geq t_{0}$ be, for any $\theta \in(0,1)$, there exists $T_{\theta} \geq t_{0}$ such that $u(\sigma(t)) \geq \theta \frac{\sigma(t)}{t} u(t), t \geq T_{\theta}$

Lemma 2.4 (LIN, W. X., 2017).Let $u(t)>0, u^{\prime}(t)>0, u^{\prime \prime}(t)>0, u^{\prime \prime \prime}(t) \leq 0, t \geq T_{\theta}$, then exists $\gamma \in(0,1)$ and $T_{\gamma} \geq T_{\theta}$, such that $u(t) \geq \gamma u^{\prime}(t), t \geq T_{\gamma}$

\section{Main Results}

In order to take advantage of the Philos-type integral averaging technique, a function $\mathrm{F}$

Let $D=\left\{(t, s) \mid t \geq s \geq t_{0}\right\}, \quad D_{0}=\left\{(t, s) \mid t>s \geq t_{0}\right\}$.

The function $H(t, s) \in C(D, R)$ belongs to the F class, and it is written as $H(t, s) \in F$, if

(i) $H(t, t)=0, t \geq t_{0} ; H(t, s)>0,(t, s) \in D_{0}$.;

(ii) $\frac{\partial H(t, t)}{\partial s}=0, t \geq t_{0} ; \frac{\partial H(t, s)}{\partial s} \leq 0,(t, s) \in D$

And continuous on D, there is a function $h \in C\left(D_{0}, R\right), \rho \in C^{1}\left(\left[t_{0}, \infty\right),(0, \infty)\right)$, which satisfies

$\frac{\partial H(t, s)}{\partial s}+A_{1}(t) H(t, s)=-h(t, s) H^{\frac{k}{k+1}}(t, s), \quad k=\min \{\alpha, \beta\}$.

Use tokens: For $\rho, \sigma \in C^{1}\left(\left[t_{0}, \infty\right),(0, \infty)\right)$, let 
$A_{1}(t)=\frac{\rho^{\prime}(t)}{\rho(t)}, \quad A_{2}(t)=q(t)(1-p)^{\alpha} \quad, \quad A_{3}(t)=q(t)(1-p)^{\beta}, \quad A(t)=q(t)\left[(1-p) \frac{\gamma \theta \sigma^{2}(t)}{t}\right]^{\beta}, \quad \varphi(t)=\int_{t}^{\infty} r^{-\frac{1}{\alpha}}(s) d s$, $R(t)=r^{\frac{\alpha+1-\beta}{\alpha}}(t), \quad L=\frac{1}{\left(Z^{\prime}(T)^{\beta-\alpha}\right.}, \quad M=\frac{1}{\left(Z^{\prime \prime}(T)\right)^{\frac{\alpha}{\beta}-1}}$, Sufficiently large for t.

Theorem 3.1 If a function $\rho \in C^{1}\left(\left[t_{0}, \infty\right),(0, \infty)\right)$ exists that satisfies $A_{1}(t)>0$, and

$$
\limsup _{t \rightarrow \infty} \int_{t_{0}}^{t}\left[\rho(s) A(s)-\frac{r(s) \rho(s)}{M^{\beta}}\left(\frac{A_{1}(s)}{\beta+1}\right)^{\beta+1}\right] d s=\infty .
$$

And

$$
\limsup _{t \rightarrow \infty} \int_{t_{0}}^{t}\left[\varphi^{\beta}(s) A_{3}(s)-\left(\frac{\beta}{\beta+1}\right)^{\beta+1} \frac{R(s)}{M^{\beta} \varphi(s)}\right] d s=\infty .
$$

Then the equation $(E)$ is oscillatory.

Proof Let the equation $(E)$ have a non-vibratory solution. If $Z(t)$ is of type (A), that is $Z(t)>0, Z^{\prime}(t)>0, Z^{\prime \prime}(t)>0$, then have

$$
x(t)=Z(t)-p(t) x(\tau(t)) \geq Z(t)-p(t) Z(\tau(t)) \geq(1-p) Z(t),
$$

from equation $(E)$, obtain

$$
\left(r(t)\left(Z^{\prime \prime}(t)\right)^{\alpha}\right)^{\prime}=-q(t) x^{\beta}(\sigma(t)) \leq-q(t)(1-p)^{\beta} Z^{\beta}(\sigma(t)) .
$$

that is

$$
\left(r(t)\left(Z^{\prime \prime}(t)\right)^{\alpha}\right)^{\prime}=-q(t) x^{\beta}(\sigma(t)) \leq-q(t)(1-p)^{\beta} Z^{\beta}(\sigma(t)) .
$$

Consider Riccati transform

$$
W(t)=\rho(t) r(t) \frac{\left(Z^{\prime \prime}(t)\right)^{\alpha}}{\left(Z^{\prime}(t)\right)^{\beta}}>0, \quad t \geq t_{2}
$$

Differentiate $t$ on both sides of the above formula, and use formula (3.3) to get

$$
\begin{aligned}
W^{\prime}(t) & =\frac{\rho^{\prime}(t)}{\rho(t)} W(t)+\rho(t)\left(\frac{r(t)\left(Z^{\prime \prime}(t)\right)^{\alpha}}{\left(Z^{\prime}(t)\right)^{\beta}}\right)^{\prime} \\
& =\frac{\rho^{\prime}(t)}{\rho(t)} W(t)+\rho(t) \frac{\left(r(t)\left(Z^{\prime \prime}(t)\right)^{\alpha}\right)^{\prime}}{\left(Z^{\prime}(t)\right)^{\beta}}-\beta \rho(t) r(t) \frac{\left(Z^{\prime \prime}(t)\right)^{\alpha+1}}{\left(Z^{\prime}(t)\right)^{\beta+1}} \\
\leq \frac{\rho^{\prime}(t)}{\rho(t)} W(t) & -\rho(t) q(t)(1-p)^{\beta} \frac{Z^{\beta}(\sigma(t))}{\left(Z^{\prime}(t)\right)^{\beta}}-\beta \frac{1}{(\rho(t) r(t))^{\frac{1}{\beta}}\left(Z^{\prime \prime}(t)\right)^{\frac{\alpha}{\beta}-1}} W^{1+\frac{1}{\beta}}(t) .
\end{aligned}
$$

Because $\left(\frac{1}{Z^{\prime \prime}(t)}\right)^{\prime}=-\frac{Z^{\prime \prime \prime}(t)}{\left(Z^{\prime \prime}(t)\right)^{2}} \geq 0$, and so $\frac{1}{Z^{\prime \prime}(t)}$ monotonically increases, also exists $T=\max \left\{t_{2}, T_{\gamma}\right\}$, When $t \geq T$, $\frac{1}{Z^{\prime \prime}(t)} \geq \frac{1}{Z^{\prime \prime}(T)}$. Since $\alpha>\beta>0$, so $\frac{\alpha}{\beta}-1>0$, we get $\left(\frac{1}{Z^{\prime \prime}(t)}\right)^{\frac{\alpha}{\beta}-1} \geq\left(\frac{1}{Z^{\prime \prime}(T)}\right)^{\frac{\alpha}{\beta}-1}=M$.

From Lemma 2.3, let $u(t)=Z^{\prime}(t)$, for any $\theta \in(0,1)$, exist $T_{\theta} \geq t_{0}$, such that 


$$
\frac{1}{Z^{\prime}(t)} \geq \theta \frac{\sigma(t)}{t} \frac{1}{Z^{\prime}(\sigma(t))}, \quad t \geq T_{\theta}
$$

From Lemma 2.4, exist $\gamma \in(0,1)$ and $T_{\gamma} \geq T_{\theta}$, such that

$$
Z(\sigma(t)) \geq \gamma \sigma(t) Z^{\prime}(\sigma(t)), \quad t \geq T \gamma
$$

So (3.4) becomes

$$
W^{\prime}(t) \leq \frac{\rho^{\prime}(t)}{\rho(t)} W(t)-\rho(t) A(t)-\frac{\beta \mathrm{M}}{(\rho(t) r(t))^{\frac{1}{\beta}}} W^{1+\frac{1}{\beta}}(t)
$$

From Lemma 2.2, let $B=\frac{\rho^{\prime}(t)}{\rho(t)}>0, A=\frac{\beta M}{(r(t) \rho(t))^{\frac{1}{\beta}}}>0, u=W(t)$, then

$$
B u-A u^{\frac{1+\beta}{\beta}} \leq \frac{1}{(\beta+1)^{\beta+1}}\left(\frac{\rho^{\prime}(t)}{\rho(t)}\right)^{\beta+1} \frac{r(t) \rho(t)}{M^{\beta}}=\frac{r(t) \rho(t)}{M^{\beta}}\left(\frac{A_{1}(t)}{\beta+1}\right)^{\beta+1} .
$$

So (3.5) becomes $W^{\prime}(t) \leq-\rho(t) A(t)+\frac{r(t) \rho(t)}{M^{\beta}}\left(\frac{A_{1}(t)}{\beta+1}\right)^{\beta+1}$.

Integrating from $\mathrm{T}$ to $\mathrm{t}$ on both sides of the above formula, we get

$$
W(t) \leq W(T)-\int_{T}^{t}\left[\rho(s) A(s)-\frac{r(s) \rho(s)}{M^{\beta}}\left(\frac{A_{1}(s)}{\beta+1}\right)^{\beta+1}\right] d s .
$$

Let $t \rightarrow \infty$, then $W(t) \rightarrow-\infty$, which contradicts $W(t)>0$, so the assumption does not hold,that is, $x(t)$ is the oscillatory solution of equation $(E)$.

If type (B) is satisfied, that is $Z(t)>0, Z^{\prime}(t)<0, Z^{\prime \prime}(t)>0$.

Because $\left(r(t)\left(Z^{\prime \prime}(t)\right)^{\alpha}\right)^{\prime}=-q(t)\left(x(\sigma(t))^{\beta} \leq 0\right.$, so $-\left(r(t)\left(Z^{\prime \prime}(t)\right)^{\alpha}\right)^{\prime} \geq 0$, and known by $q(t)>0,(1-p)>0, Z^{\prime}(t)<0$, which is $q(t)(1-p)^{\beta}\left(Z^{\prime}(t)\right)^{\beta}<0$, so there is

$$
-\left(r(t)\left(Z^{\prime \prime}(t)\right)^{\alpha}\right)^{\prime} \geq q(t)(1-p)^{\beta}\left(Z^{\prime}(t)\right)^{\beta}=A_{3}(t)\left(Z^{\prime}(t)\right)^{\beta} .
$$

Consider Riccati transform

$$
U(t)=-\frac{r(t)\left(Z^{\prime \prime}(t)\right)^{\alpha}}{\left(Z^{\prime}(t)\right)^{\beta}}>0, t \geq t_{2},
$$

differentiate $\mathrm{T}$ in equation (3.7) and use equations (3.6) and (3.7) to get

$$
\begin{aligned}
U^{\prime}(t) & =\frac{-\left(r(t)\left(Z^{\prime \prime}(t)\right)^{\alpha}\right)^{\prime}}{\left(Z^{\prime}(t)\right)^{\beta}}+\beta \frac{r(t)\left(Z^{\prime \prime}(t)\right)^{\alpha+1}}{\left(Z^{\prime}(t)\right)^{\beta+1}} \\
& \geq A_{3}(t)+\beta \frac{1}{r^{\frac{1}{\beta}}(t)\left(Z^{\prime \prime}(t)\right)^{\frac{\alpha}{\beta}-1}} U^{1+\frac{1}{\beta}}(t) .
\end{aligned}
$$

$\operatorname{Because}\left(\frac{1}{Z^{\prime \prime}(t)}\right)^{\prime}=-\frac{Z^{\prime \prime \prime}(t)}{\left(Z^{\prime \prime}(t)\right)^{2}} \geq 0$, and so $\frac{1}{Z^{\prime \prime}(t)}$ monotonically increases, existence $T=\max \left\{t_{2}, T_{\gamma}\right\}$, 
when $t \geq T, \frac{1}{Z^{\prime \prime}(t)} \geq \frac{1}{Z^{\prime \prime}(T)}$. Since $\alpha>\beta>0$, so $\frac{\alpha}{\beta}-1>0$, we get $\left(\frac{1}{Z^{\prime \prime}(t)}\right)^{\frac{\alpha}{\beta}-1} \geq\left(\frac{1}{Z^{\prime \prime}(T)}\right)^{\frac{\alpha}{\beta}-1}=M$,

then from (3.8)

$$
U^{\prime}(t) \geq A_{3}(t)+\frac{\beta M}{r^{\frac{1}{\beta}}(t)} U^{1+\frac{1}{\beta}}(t) .
$$

Multiply both sides of formula (3.9) by $\varphi^{\beta}(t)$, and integrate from $T$ to, get

$$
\begin{aligned}
& \int_{T}^{t} \varphi^{\beta}(s) A_{3}(s) d s \leq \int_{T}^{t} \varphi^{\beta}(s) U^{\prime}(s) d s-\int_{T}^{t} \beta M \varphi^{\beta}(s) r^{-\frac{1}{\beta}}(s) U^{1+\frac{1}{\beta}}(s) d s \\
& \quad \leq \varphi^{\beta}(t) U(t)+\int_{T}^{t}\left[\beta \varphi^{\beta-1}(s)\left(r^{-\frac{1}{\alpha}}(s) U(s)-M \varphi(s) r^{-\frac{1}{\beta}}(s) U^{1+\frac{1}{\beta}}(s)\right)\right] d s .
\end{aligned}
$$

Using Lemma 2.2, take $B=r^{-\frac{1}{\alpha}}(s)>0, A=M \varphi(s) r^{-\frac{1}{\beta}}(s)>0, u=U(s)$, then

$$
B u-A u^{\frac{\beta+1}{\beta}} \leq \frac{\beta^{\beta}}{(\beta+1)^{\beta+1}} \frac{R(s)}{M^{\beta} \varphi^{\beta}(s)} .
$$

Therefore from (3.10), we obtain

$$
\int_{T}^{t}\left[\varphi^{\beta}(s) A_{3}(s)-\left(\frac{\beta}{\beta+1}\right)^{\beta+1} \frac{R(s)}{M^{\beta} \varphi(s)}\right] d s \leq \varphi^{\beta}(t) U(t) .
$$

Since $\left(r(t)\left(Z^{\prime \prime}(t)\right)^{\alpha}\right)^{\prime} \leq 0$, then when $s>t$, there is $r(s)\left(Z^{\prime \prime}(s)\right)^{\alpha} \leq r(t)\left(Z^{\prime \prime}(t)\right)^{\alpha}$, that is,

$$
Z^{\prime \prime}(s) \leq r^{\frac{1}{\alpha}}(t) Z^{\prime \prime}(t)\left(\frac{1}{r(s)}\right)^{\frac{1}{\alpha}} \text {. }
$$

Integrate s from t to $l(l>s)$ on both sides (3.12) to get

$$
0<Z^{\prime}(l)-Z^{\prime}(t) \leq r^{\frac{1}{\alpha}}(t) Z^{\prime \prime}(t) \int_{t}^{l}\left(\frac{1}{r(s)}\right)^{\frac{1}{\alpha}} d s .
$$

Therefore $0<-Z^{\prime}(t) \leq-Z^{\prime}(l)+r^{\frac{1}{\alpha}}(t) Z^{\prime \prime}(t) \int_{t}^{l}\left(\frac{1}{r(s)}\right)^{\frac{1}{\alpha}} d s$, that is,

$$
r^{\frac{1}{\alpha}}(t) Z^{\prime \prime}(t) \int_{t}^{l}\left(\frac{1}{r(s)}\right)^{\frac{1}{\alpha}} d s>Z^{\prime}(l)>Z^{\prime}(t) .
$$

Because $\varphi(t)=\int_{t}^{\infty}\left(\frac{1}{r(s)}\right)^{\frac{1}{\alpha}} d s$, let $l \rightarrow \infty$, then from (3.13), we get

$$
\varphi^{\beta}(t) U(t) \leq-r^{1-\frac{\beta}{\alpha}}(t)\left(Z^{\prime \prime}(t)\right)^{\alpha-\beta} \leq 0,
$$

and because $\varphi^{\beta}(t) U(t) \geq 0$, so $\varphi^{\beta}(t) U(t)=$, so have 


$$
\int_{T}^{t}\left[\varphi^{\beta}(s) A_{3}(s)-\left(\frac{\beta}{\beta+1}\right)^{\beta+1} \frac{R(s)}{M^{\beta} \varphi(s)}\right] d s \leq \varphi^{\beta}(t) U(t)=0 .
$$

This contradicts (3.2), so the assumption is not true. That is, when $Z(t)$ satisfies (B), $x(t)$ is the vibrational solution of equation $(E)$. The proof is complete.

Theorem 3.2. Let there be a function $\rho \in C^{1}\left(\left[t_{0}, \infty\right),(0, \infty)\right)$ such that $(3.1)$ holds, and satisfies

$$
\lim _{t \rightarrow \infty} \int_{t_{0}}^{t} A_{3}(s) d s=\infty .
$$

Then, equation $(E)$ is oscillatory.

Proof Let $x(t)$ be the non-vibration solution of the equation $(E)$, similar to the proof of Theorem 3.1, if $Z(t)$ is of type (A), that is, here, the proof process is the same as the proof process of the first part of Theorem 3.1. That is, when $Z(t)$ satisfying (A) type, $x(t)$ is the oscillate solution of the equation.

If $Z(t)$ satisfies type (B), that is $Z(t)>0, Z^{\prime}(t)<0, Z^{\prime \prime}(t)>0$,

Since $\left(r(t)\left(Z^{\prime \prime}(t)\right)^{\alpha}\right)^{\prime}=-q(t)\left(x(\sigma(t))^{\beta} \leq 0\right.$, and $\left(A_{1}\right)$ know $q(t)>0,(1-p)>0, Z^{\prime}(t)<0$, we get $q(t)(1-p)^{\beta}\left(Z^{\prime}(t)\right)^{\beta}<0$, and we have

$$
\left(r(t)\left(Z^{\prime \prime}(t)\right)^{\alpha}\right)^{\prime} \leq-q(t)(1-p)^{\beta}\left(Z^{\prime}(t)\right)^{\beta}=-A_{3}(t)\left(Z^{\prime}(t)\right)^{\beta}
$$

Define Riccati function as follows

$$
U_{1}(t)=\frac{r(t)\left(Z^{\prime \prime}(t)\right)^{\alpha}}{\left(Z^{\prime}(t)\right)^{\beta}}<0, t \geq t_{2},
$$

derivate $t$ on both sides of (3.16) and use the result of (3.15) to get

$$
\begin{aligned}
U_{1}^{\prime}(t) & =\frac{\left(r(t)\left(Z^{\prime \prime}(t)\right)^{\alpha}\right)^{\prime}}{\left(Z^{\prime}(t)\right)^{\beta}}-\beta \frac{r(t)\left(Z^{\prime \prime}(t)\right)^{\alpha+1}}{\left(Z^{\prime}(t)\right)^{\beta+1}} \\
& \leq-A_{3}(t)-\beta \frac{r(t)\left(Z^{\prime \prime}(t)\right)^{\alpha+1}}{\left(Z^{\prime}(t)\right)^{\beta+1}} .
\end{aligned}
$$

Since $\beta>0$ is the ratio of two positive and odd numbers, so $\beta+1$ is an even number, that is, $\beta \frac{r(t)\left(Z^{\prime \prime}(t)\right)^{\alpha+1}}{\left(Z^{\prime}(t)\right)^{\beta+1}}>0$, and then (3.17) becomes

$$
U_{1}^{\prime}(t) \leq-A_{3}(t)
$$

Integrate both sides of (3.18) from $T$ to $t$ to get

$$
U_{1}\left(t_{2}\right) \geq U_{1}(t)+\int_{t_{2}}^{t} A_{3}(s) d s
$$

Let $t \rightarrow \infty$, according to (3.14), obtain $U_{1}\left(t_{2}\right) \rightarrow+\infty$, which contradicts $U_{1}(t)<0$, so the assumption is not true. Thus, when (B) is satisfied, $x(t)$ is the oscillatory solution of the equation $(E)$. The proof is complete.

Corollary3.3. Let function $\rho \in C^{1}\left(\left[t_{0}, \infty\right),(0, \infty)\right)$ and function $H(t, s) \in F$ exist so that (3.14) holds, and

$$
\limsup _{t \rightarrow \infty} \frac{1}{H\left(t, t_{0}\right)} \int_{t_{0}}^{t}\left[H(t, s) \rho(s) A(s)-\frac{\rho(s) r(s)}{M^{\beta}}\left(\frac{|h(t, s)|}{\beta+1}\right)^{\beta+1}\right] d s=+\infty .
$$

Then equation $(E)$ is oscillatory.

\section{Application}

Example: Consider a third-order neutral differential equation

$$
\left[t^{6}\left(\left(x(t)+\frac{1}{3} x(t)\right)^{\prime \prime}\right)^{3}\right]^{\prime}+t^{9} x(t)=0, t \geq t_{0}>m>0,
$$


here we take $\alpha=3, \beta=1, r(t)=t^{6}, q(t)=t^{9}, p(t)=\frac{1}{3}, p=\frac{1}{2}, \tau(t)=t, \sigma(t)=t$,

$\rho(t)=t$, from Lemma 3, when $\sigma(t)=t, \quad \theta=1, \gamma \in(0,1), m=\max \left\{\frac{1}{2 M}, \sqrt[6]{\frac{1}{2 M \gamma}}\right\}$,obviously there

$R(t)=r^{\frac{\alpha+1-\beta}{\alpha}}(t)=r(t)=t^{6}, \varphi(t)=\int_{t}^{\infty} r^{-\frac{1}{\alpha}}(s) d s=\int_{t}^{\infty} \frac{1}{s^{2}} d s=\frac{1}{t}, A_{1}(t)=\frac{\rho^{\prime}(t)}{\rho(t)}=\frac{1}{t}>0, \quad A_{3}(t)=q(t)(1-p)^{\beta}=\frac{1}{2} t^{10}$,

$A(t)=q(t)\left[(1-p) \frac{\gamma \theta \sigma^{2}(t)}{t}\right]^{\beta}=t^{9} \times \frac{1}{2} \times \frac{\gamma t^{2}}{t}=\frac{\gamma}{2} t^{10}$

So get

$$
\begin{aligned}
& \limsup _{t \rightarrow \infty} \int_{t_{0}}^{t}\left[\rho(s) A(s)-\frac{r(s) \rho(s)}{M^{\beta}}\left(\frac{A_{1}(s)}{\beta+1}\right)^{\beta+1}\right] d s \\
= & \limsup _{t \rightarrow \infty} \int_{t_{0}}^{t}\left[s \times \frac{\gamma^{10}}{2}-\frac{s^{6} \times s}{M}\left(\frac{1}{2 s}\right)^{2}\right] d s=\limsup _{t \rightarrow \infty} \int_{t_{0}}^{t}\left(\frac{t^{5}\left(2 M t^{6}-1\right)}{4 M}\right)=\infty . \\
& \limsup _{t \rightarrow \infty} \int_{t_{0}}^{t}\left[\varphi^{\beta}(t) A_{3}(t)-\left(\frac{\beta}{\beta+1}\right)^{\beta+1} \frac{R(s)}{M^{\beta} \varphi(s)}\right] d s \\
= & \limsup _{t \rightarrow \infty} \int_{t_{0}}^{t}\left(\frac{1}{t} \times \frac{t^{9}}{2}-\left(\frac{1}{2}\right)^{2} \frac{t^{7}}{M}\right) d s=\limsup _{t \rightarrow \infty} \int_{t_{0}}^{t} \frac{t^{7}(2 M t-1)}{4 M} d s=\infty . \\
& \lim _{t \rightarrow \infty} \int_{t_{0}}^{t} A_{3}(s) d s=\lim _{t \rightarrow \infty} \int_{t_{0}}^{t} \frac{s^{9}}{2} d s=\lim _{t \rightarrow \infty}\left(\frac{t^{10}}{20}-\frac{t_{0}^{10}}{20}\right)=\infty .
\end{aligned}
$$

Obviously Equation $\left(E_{2}\right)$ satisfies the conditions (3.1) and (3.2) of Theorem 3.1, and it satisfies the conditions (3.14) of Theorem 3.2. So that equation $\left(E_{2}\right)$ is oscillatory.

Mark: Our results improve or generalize the results of some literature. The results of reference (LI., et al, 2017).) cannot be applied to this example.

\section{Acknowledgments}

Maoming Science and Technology Plan Project of Guangdong Province (2015038), Key Project of Scientific Research Support Fund of Guangdong University of Petrochemical Technology (KY2018001) and Guangdong University Student Innovation and Entrepreneurship Project

\section{References}

Dzurina, J. O. Z. E. F., \& Baculikov, B. L. A. N. K. A. (2012). Oscillation Of Third-Order Quasi-Linear Advanced Differential Equations. Differ. Equ. Appl., 4(3), 411-421. https://doi.org/10.7153/dea-04-23

Hui, Y. X., \& Wang, J. J. (2017). Oscillatory results of Third-Order Neutral Semi-linear Neutral Differential Equations with Delay Argument. Journal of Jinggangshan University, 38(1), 8-13.

Li, Q., Yang, J., Li, X., \& Lin, Q. (2017). Oscillate Criteria of Third Order Semi-Linear Neutral Differential Equations with Delay Argument. https://doi.org/10.12677/PM.2017.74045

Li, T. X., Han, Z. L., Zhang, C. H., \& Sun, Y. (2012). Oscillation criteria for third-order Emden-Fowler delay dynamic equations on time scales. Acta Math. Sci., Ser. A, 32, 222-232.

Li, Y. D., Gao, Z. H., \& Deng, Y. H. (2012). Oscillation for Third Order Semi-linear Neutral Differential Equations. Journal of Beihua University (Natural Science), (3), 5.

Lin, Q.W., \& Yu, Y. H. (2015). Oscillate Criteria of Third Order Semi-Linear Neutral Differential Equations with Delay Argument. J. Sys. Sci. \& Math. Scis., 35(2), 233-244.

Liu, H., Meng, F., \& Liu, P. (2012). Oscillation and asymptotic analysis on a new generalized Emden-Fowler equation. Applied Mathematics and computation, 219(5), 2739-2748. https://doi.org/10.1016/j.amc.2012.08.106 
Luo, L. P., Yu, Y. H., \& Luo, Z. G. (2016). Oscillation Analysis of Third Order Nonlinear Neutral Differential Equations. Journal of Systems Science and Mathematical Sciences, 36(4), 551-559.

Qin, G., Huang, C., Xie, Y., \& Wen, F. (2013). Asymptotic behavior for third-order quasi-linear differential equations. Advances in Difference equations, 2013(1), 305. https://doi.org/10.1186/1687-1847-2013-305

Su, X. X., Dai, L. N., Wu, S. M., \& Lin, Q. W. (2017). Oscillation of Second-Order Semilinear Differential Equations. Advances in Applied Mathematics, 6(3), 417-422. https://doi.org/10.12677/AAM.2017.63048

Wen-xian, L. I. N. (2017). Oscillation of certain third-order half linear neutral functional differential equations with damping. Journal of East China Normal University (Natural Science), (3), 48.

Wu, S., Lin, J., Li, Q., \& Lin, Q. (2019). Oscillation of a Class of Third Order Semi-Linear Neutral Delay Differential Equations.

ZENG, Y. H., \& Yu, Y. H. (2014). Oscillation for third order half-linear delay differential equations. J. Sys. Sci. \& Math. Scis., 34(2), 231-237.

Zeng, Y. H., Luo, L. P., \& Yu, Y. H. (2015). Oscillation of a Class of Third Order Semi-Linear Neutral Delay Differential Equations. Advances in Applied Mathematics, 8(03), 473.

Zhang, Z. Y., Wang, X. X., \& Yu, Y. H. (2015). On Comparison Criteria for Oscillation of Third Order Nonlinear Functional Differential Equations. Mathematica Applicata, 38(3), 450-459.

\section{Copyrights}

Copyright for this article is retained by the author(s), with first publication rights granted to the journal.

This is an open-access article distributed under the terms and conditions of the Creative Commons Attribution license (http://creativecommons.org/licenses/by/4.0/). 